\title{
Interaction of Mitoxantrone-Loaded Cholesterol Modified Pullulan Nanoparticles with Human Serum Albumin and Effect on Drug Release
}

\author{
Liming Yuan, ${ }^{1}$ Bu Guo, ${ }^{1}$ Wu Zhong, ${ }^{1}$ Yu Nie, ${ }^{1}$ Xiaoyan Yao, ${ }^{1}$ Xiaofeng Peng, ${ }^{1}$ Rong Wang, \\ Hongyuan Yu, ${ }^{1}$ Shanyi Yang, ${ }^{1}$ Chunlian He, ${ }^{1}$ Xiaojun Tao $\mathbb{D}^{1,2}$ and Qiufang Zhang $\mathbb{D}^{2}$ \\ ${ }^{1}$ Key Laboratory of Study and Discovery of Small Targeted Molecules of Hunan Province, School of Medicine, \\ Hunan Normal University, Changsha 410013, China \\ ${ }^{2}$ Department of Pharmacology, Hubei University of Medicine, Shiyan, Hubei 442000, China
}

Correspondence should be addressed to Xiaojun Tao; xiaojtao@126.com and Qiufang Zhang; zqf1112000@163.com

Received 10 December 2018; Revised 17 March 2019; Accepted 15 April 2019; Published 16 May 2019

Academic Editor: Surinder Singh

Copyright ( 2019 Liming Yuan et al. This is an open access article distributed under the Creative Commons Attribution License, which permits unrestricted use, distribution, and reproduction in any medium, provided the original work is properly cited.

\begin{abstract}
To clarify nanoparticle-protein interaction and their action characteristics, the interactions between MTO-CHP NPs and human serum albumin (HSA) were studied by isothermal titration calorimetry (ITC), fluorescence spectroscopy, dynamic light scattering (DLS), and circular dichroism spectroscopy (CD). Hydrophobically modified pullulan (CHP) nanoparticles (NPs) loaded with mitoxantrone (MTO) were prepared (MTO-CHP NPs) with size $166.9 \mathrm{~nm}$. The spherical shape was verified by transmission electron microscopy (TEM). The ITC results demonstrated an interaction between MTO-CHP NPs mainly by hydrophobic interaction force, electrostatic force, and hydrogen bonding. The mean binding constant $K_{\mathrm{A}}$ was $0.832 \times 10^{4} \mathrm{M}^{-1}$ and mean HSA coverage $0.939 \pm 0.302$. MTO-CHP NPs could quench the fluorescence intensity of HSA, which gradually decreased to be balanced in $9 \mathrm{~h}$ and indicated the completion of the complexation. The size and zeta potential changes of the combined particle were dynamically detected with DLS at $0,3,6,9,12,15$, and $18 \mathrm{~h}$. When the reaction was completed at $9 \mathrm{~h}$, the particle size and potential remained stable, accompanied by a size change from 89.91 to about $145 \mathrm{~nm}$ and potential change from -15 to $-3 \mathrm{mV}$, respectively. The results of CD measurement showed that the change in ellipticity of HSA at $208 \mathrm{~nm}$ was similar to the fluorescence spectra and DLS measurements with MTO-CHP NPs combined with HSA. At the beginning of the reaction, the proportion of $\alpha$-helix was $52.3 \%$ to $43.7 \%$, which decreased by $39.1 \%$ at compound stabilization. The release of MTO from MTO-CHP NPs at $\mathrm{pH}=5.6$ was significantly accelerated, whereas that of MTO from HSA-MTO-CHP NPs was significantly reduced, and the drug release was significantly slowed down even under acidic conditions, which indicates the beneficial effect of HSA on the persistence and stability of the HSA-MTO-CHP NP compound.
\end{abstract}

\section{Introduction}

Nanopreparations have good sustained release and targeting and are a hot topic of research in drug delivery systems $[1,2]$. The nanometer size can be used to passively target tumor tissue via an enhanced permeability and retention effect, achieve local fixed-point release, and exert an antitumor effect [3]. Nanopreparations enter the blood circulation by intravenous injection and are susceptible to adsorption of plasma proteins before passively targeting tumor tissues [4]. The adsorption of nanoparticles (NPs) by plasma proteins changes the characteristics of the NPs, such as size, surface charge, and surface hydrophobicity, which alters their biological behaviors in vivo and thus their efficacy $[5,6]$. The type and quantity of plasma protein adsorption are closely related to the nature of the NPs themselves. The surface of NPs mainly adsorbs high-abundance proteins and proteins with strong adsorption to NPs $[7,8]$.

Human serum albumin (HSA) contains more than 60\% high-abundance proteins in serum. HSA has three domains I, II, and III-each with two subdomains, A and B [9]. It can bind to various endogenous and exogenous compounds 
via amino acid residues and mainly binds to hydrophobic cavities at $\mathrm{II}_{\mathrm{A}}$ (site I) and $\mathrm{III}_{\mathrm{A}}$ (site II) [10]. HSA binding is an important factor in helping to transport drugs and limiting their free active concentrations [11, 12]. Many studies have investigated the reaction of small molecule drugs and macromolecular drugs with HSA. Small-molecule drugs such as palmatine react with HSA, which will affect the tissue distribution, metabolism, and effect of palmatine [9]. Macromolecular substances such as fluorescent carbon dots (CDs) of novel carbon-based nanomaterials react with HSA, affecting the actual medical application of CDs and weakening the biological activity of HSA [13].

Recently, some HSA NPs as a new drug delivery systems show potential clinical value, such as targeting tumor or inflamed tissues with high stability, high biodegradability, low toxicity, and immunogenicity [14]. Zhang et al. used amphiphilic poly(N-(2-hydroxypropyl)-methacrylamide) copolymer NPs (pHPMA NPs) loaded with doxorubicin (DOX) or pHPMA NPs without DOX in the HSA solution under a physiological environment [15]. Unlike unloaded NPs, drug-loaded NPs did not interact with HSA, so the delivery of DOX by using pHPMA NPs in the human body is not affected by the presence of HSA [15]. However, the interaction of HSA with drug-loaded NPs may affect the targeting and sustained drug release properties. For example, morin $\left(3,5,7,2^{\prime}, 4^{\prime}\right.$-pentahydroxyflavone)-loaded poly(lacticco-glycolic acid) (PLGA) NPs (MPNPs) combined with HSA could promote morin's continuous release [16]. Therefore, the study of the interaction between drug-loaded NPs and HSA is of great significance to the research and optimization of drug-loaded nanopreparations.

In this study, we used cholesteric modified pullulan (CHP) NPs loaded with mitoxantrone (MTO) as drugloaded NPs to explore the interaction with HSA. MTO is an anthracycline antibiotic that has been used as an antitumor drug in clinical practice. It has no selectivity for tumor cells and has a large toxic side effect in clinical application. It can be loaded into the hydrophobic center of polymer NPs, which can passively target tumor cells via the enhanced permeability and retention effect, reduce the uptake of drugs by normal tissues, and enhance the efficacy and safety [17]. CHP polymer NPs can self-assemble to form NPs in water consisting of a hydrophobic core and a hydrophilic shell [18]. The hydrophobic core can encapsulate the hydrophobic substance, and the hydrophilic outer shell can enhance the solubility and stability in the aqueous solution. CHP polymer NPs are nontoxic and have good biocompatibility; they can be degraded by amylase in the body. So, they are often used as a nanomaterial to load drugs in biological applications; examples are polytoluene and 5-fluorouracil [19-21]. In recent years, many studies have investigated the use of CHP and its derivatives as nanotargeted antitumor drug carriers, but few studies have examined the interaction between drug-loaded CHP and protein and its biological effects.

MTO and HSA are combined by hydrophobic interaction, hydrogen bond, and electrostatic interaction, mainly taking place in the hydrophobic cavity of HSA. This is a rapid and reversible process that quickly reaches dynamic equilibrium [22]. CHP forms complex NPs, embedding various proteins into the NP core, rather than adsorbing on the NP surface [7]. Adding HSA into MTO-containing CHP NPs could significantly slow down the release rate of MTO [7].

In the present study, we investigated the specific interactions between MTO, CHP NPs, drug-loaded CHP NPs, and HSA by using ITC, fluorescence spectroscopy, DLS, and circular dichroism (CD) spectroscopy. We further revealed the interaction characteristics between drug-loaded CHP NPs and HSA, thereby elucidating the effect of the HSA complex on drug release of drug-loaded CHP NPs. These results will help in the exploration and development of clinically meaningful HSA NPs.

\section{Materials and Methods}

2.1. Materials. HSA (molecular weight $66.5 \mathrm{kDa}$ ) was from Sigma-Aldrich (St. Louis, MO, USA). HSA solution was prepared in $10 \mathrm{mM}$ phosphate buffer at $\mathrm{pH}$ 7.4. Pullulan (average molecular weight $200 \mathrm{kDa}$, degree of deacetylation $>90 \%$ ) was from Solarbio (Beijing) and mitoxantrone hydrochloride from Sigma-Aldrich (Shanghai). Other chemical reagents were of analytical grade and were from Changsha Huicheng (Changsha, China).

\subsection{Methods}

2.2.1. Synthesis of Cholesteric Hydrophobically Modified Pullulan (CHP) and Its Characterization by Fourier Transform Infrared (FT-IR) Spectroscopy. The synthesis of CHP conjugate was previously reported [23]. The FT-IR spectra of pullulan, cholesterol succinate (CHS), and CHP were obtained as $\mathrm{KBr}$ pellets for FT-IR spectroscopy (Nicolet Nexus 470-ESP, USA) at room temperature.

\subsubsection{Preparation and Characterization of Drug-Loaded NPs.} The presynthesized CHP (cholesterol substitution 4.06) was selected in the following preparation. MTO-loaded CHP NPs were prepared by a dialysis method [24]. DLS (Zetasizer $3000 \mathrm{HS}$, Malvern Instruments, Malvern, UK) was used to determine the size distribution and zeta potential of the drug-loaded NPs at $11.4 \mathrm{~V} / \mathrm{cm}, 13.0 \mathrm{~mA}$. A drop of MTOCHP NP $(1.0 \mathrm{mg} / \mathrm{mL})$ solution was placed into a copper mesh negatively stained with phosphotungstic acid (2\%), and the grid was naturally air-dried and analyzed by TEM (Tecnai G2 20 S-Twin, FEI Hong Kong, Hong Kong) observing morphological characteristics at an acceleration voltage of $80 \mathrm{kV}$.

\subsubsection{Characterization of the Interaction between HSA and Drug-Loaded NPs}

(1) Isothermal Titration Calorimetry (ITC). All solutions were degassed before titration [25]. The heat change was measured by using ITC (VIP-ITC, MicroCal, Northampton, MA, USA). The ligand was placed in a syringe, and the receptor was added to the sample cell. The buffer solution was loaded into the reference cell, and the reaction container and apparatus (the system) were maintained in a constant 
temperature $25^{\circ} \mathrm{C}$ and constant pressure to atmosphere. At the beginning of the titration, the syringe was placed in the sample cell and dripped at regular intervals to ensure balanced sample action. The thermodynamic parameters were then obtained and displayed in the curve. The raw data are expressed as thermal changes over time. Three sets of experiments were performed: the first group with $0.45 \mathrm{mM}$ MTO dropped into $0.002 \mathrm{mM}$ HSA, the second

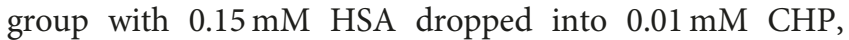
and the third group with $0.9 \mathrm{mM}$ HSA dropped into $0.01 \mathrm{mM}$ CHP-MTO. Before the curve was fitted, the first data point was always deleted from the data set, and the data were analyzed by using the Origin 8.5 software. A set of site models was used to obtain the composite stability constant $\left(K_{\mathrm{A}}\right)$ and molar reaction metamorphosis $\left(\Delta H^{\circ}\right)$. Then, the standard free energy $\left(\Delta G^{\circ}\right)$ and entropy change $\left(\Delta S^{\circ}\right)$ were calculated as $\Delta G^{\circ}=-\mathrm{RT} \ln K_{\mathrm{A}}=\Delta H^{\circ}-T \Delta S^{\circ}$. The enthalpy change and entropy change reflect the interaction mode and mechanism [26].

(2) Fluorescence Spectroscopy. HSA contains a tryptophan that is excited in the ultraviolet region to emit fluorescence, forming a fluorescent spectrum. When other molecules interact with HSA, tryptophan fluorescence may change, depending on the effect of this interaction on protein conformation $[27,28]$. HSA and MTO, CHP NPs, and MTO-CHP NPs were mixed and reacted at a molecular ratio of 3.6:1 (HSA: $1.5 \times 10^{-5} \mathrm{~mol} / \mathrm{L}$ ). The obtained mixture was placed in a $2 \mathrm{~mL}$ Eppendorf tube (EP tube) and shaken at $20 \mathrm{rpm}$, $25^{\circ} \mathrm{C}$, for $24 \mathrm{~h}$ for full reaction. Fluorescence spectrophotometry (Shimadzu RF-4500, Japan) was used to record the fluorescence intensity of free HSA and HSA that began to bind or was already bound to MTO, CHP NPs, and MTO-CHP NPs. The maximum changes in fluorescence intensity with recombination time were recorded. The tryptophan chromophore in the HSA molecule was excited at $280 \mathrm{~nm}$ [28], and the emission spectrum was recorded at $290-450 \mathrm{~nm}$. The excitation and emission slit widths were 5 and $12 \mathrm{~nm}$, respectively.

(3) Circular Dichroism (CD). In biological fluids, the secondary structure of human serum albumin may be toxic because of changes in drug-induced conformation and function. Because CD spectroscopy is sensitive to changes in protein structure [29], it was used to study the structural changes of HSA after the compound formation.

The CHP-HSA complex was prepared by storing the complex in a $2 \mathrm{~mL}$ EP tube, keeping it at a constant temperature of $25^{\circ} \mathrm{C}$ and stirring on a shaking table at $12 \mathrm{rpm}$ for $18 \mathrm{~h}$. The CD spectra (at wavelength $200-250 \mathrm{~nm}$ ) of free HSA, HSA after the addition of MTO, CHP NPs, and MTO-CHP NPs were recorded by using a CD spectrometer (Jasco J-810, Japan) at $37^{\circ} \mathrm{C}$ with a $0.1 \mathrm{~cm}$ cuvette cell. Aa, $\mathrm{Ab}, \mathrm{Ac}$, and Ad represent the CD of free HSA, HSA and MTO, HSA and CHP NPs, and HSA and MTO-CHP NPs, respectively, at the beginning of complex formation, and $\mathrm{Ba}, \mathrm{Bb}$, and $\mathrm{Bc}$ represent the $\mathrm{CD}$ of HSA and MTO, HSA and CHP NPs, and HSA and MTO-CHP NPs, respectively,

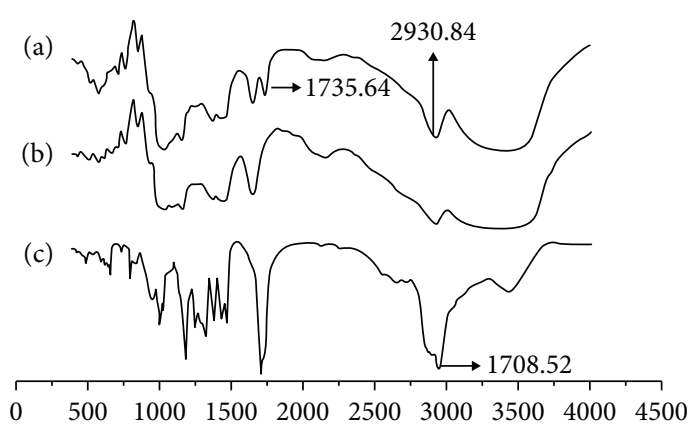

Figure 1: IR spectra of (a) CHP and (b) pullulan (c) CHS.

at the end of complex formation. The concentration of HSA in all samples was $1.0 \mathrm{mg} / \mathrm{mL}\left(1.5 \times 10^{-5} \mathrm{~mol} / \mathrm{L}\right)$. The content of the $\alpha$-helix in HSA was calculated as follows [30]:

$$
\begin{gathered}
\text { MRE }=\frac{\mathrm{CD} m \text { deg }}{c_{p} n l \times 10}, \\
\alpha-\operatorname{Helix}(\%)=\left[\frac{-\mathrm{MRE}_{208}-4000}{33000-4000}\right] \times 100,
\end{gathered}
$$

where $\mathrm{MRE}_{208}$ is the average residual ellipticity at $208 \mathrm{~nm}$ $\left(\operatorname{deg} \mathrm{cm}^{-2} \mathrm{dmol}^{-1}\right), n$ is the number of HSA amino acid residues (585), $C_{p}$ is the molar concentration of HSA $\left(1.5 \times 10^{-5} \mathrm{~mol} / \mathrm{L}\right)$, and $L$ is the length of the cuvette cell $(0.1 \mathrm{~cm})$.

2.2.4. Determining Drug Release. The standard curve of MTO was obtained by using a microplate spectrophotometer (UV-384 Plus, Molecular Devices, USA). The NPs were dialyzed in phosphate-buffered saline $(\mathrm{pH}=7.4)$ and acidic solution $(\mathrm{pH}=5.6)$ simulating the tumor microenvironment to study MTO release in vitro. In brief, the solution of CHP-NPs loaded with MTO and HSA-MTO-CHP-NPs $(2 \mathrm{mg} / \mathrm{mL})$ was placed in a VISKING dialysis tube (molecular weight cut-off $12-14 \mathrm{kDa}$, USA) and dialyzed in an air-bath shaker for $50 \mathrm{rpm}$ at $37^{\circ} \mathrm{C}$. At a predetermined time, $5 \mathrm{~mL}$ released medium was removed with a pipette and $5 \mathrm{~mL}$ fresh medium was added. MTO release was determined at $608 \mathrm{~nm}$ by UV spectrophotometry followed by calculating the cumulative release percentage $(Q \%)[31]$.

\section{Results}

3.1. FT-IR Analysis. As shown in Figure 1, CHP showed a distinct ester carbonyl absorption peak near $1735 \mathrm{~cm}^{-1}$, while the carboxyl peak near $1708 \mathrm{~cm}^{-1}$ disappeared in the infrared spectrum of CHS. The absorption peaks of methyl and methylene groups near $2930 \mathrm{~cm}^{-1}$ were significantly enhanced, indicating that $\mathrm{CHS}$ was grafted on to pullulan.

3.2. Characterization of Drug-Loaded CHP NPs. The average size distribution and polydispersity index (PDI) of MTO-CHP NPs was $166.9 \mathrm{~nm}$ and 0.213 , respectively (Figure 2(b)), so the NPs were stable and the particle size was uniform. As compared with CHP NPs $(-1.21 \mathrm{mV})$ [7], 


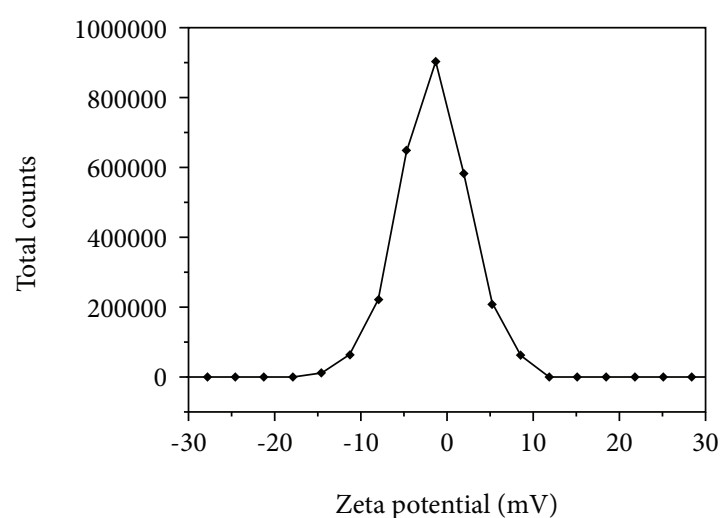

(a)

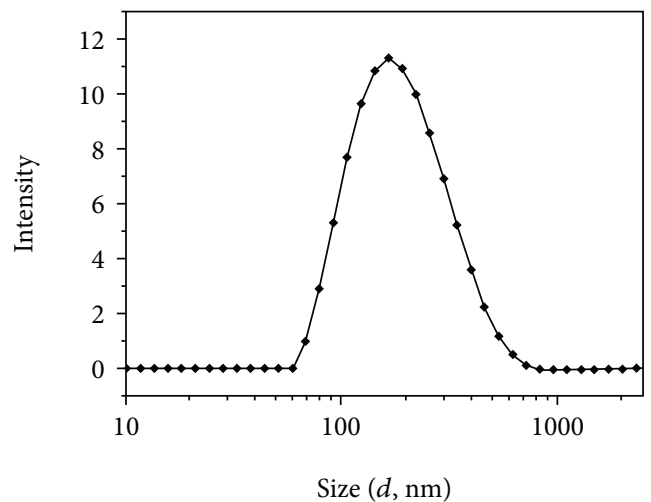

(b)

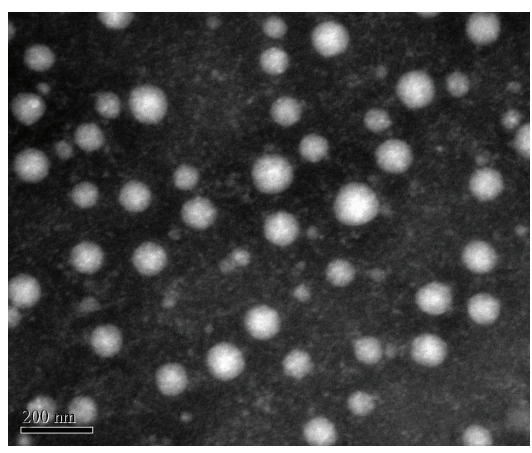

(c)

FIgURe 2: Zeta potential (a), size distribution (b), and transmission electron microscopy (TEM) image (c) of MTO-CHP NPs.

the mean zeta potential of MTO-CHP NPs was $-0.698 \pm$ $6.09(\mathrm{mV})$ and higher (Figure 2(a)), so MTO decreased the electronegativity of CHP NPs, which corresponded to the positive electrochemistry of MTO due to the presence of imino groups [32]. The TEM image shows the spherical shape of MTO-CHP NPs (Figure 2(c)).

\subsection{ITC-Determined Characterizations of Free Drugs, CHP} NPs, and Drug-Loaded CHP NPs Interacting with HSA. Figure 3(a) shows MTO titration into HSA. The lines present positive and negative peaks during the titration process. At each titration, the positive peaks were almost the same, about $0.1125 \mu \mathrm{cal} / \mathrm{sec}$, whereas the absolute value of negative peaks decreased with increasing drops from the second drop. The corresponding curve showed an increasing trend, and the dots were scattered and undulating.

Corresponding thermodynamic parameters could be obtained from the curve. According to $\triangle G^{\circ}=\triangle H^{\circ}-T \triangle S^{\circ}$, $\triangle G^{\circ}<0$ indicates that the reaction proceeded spontaneously for MTO binding HSA via hydrophobic force, electrostatic interaction, and hydrogen, and $\triangle H^{\circ}<0$ and $\triangle S^{\circ}>0$ indicate that the adsorption of MTO and HSA was mainly due to electrostatic interaction $[33,34]$. MTO is electropositive due to its imino group, whereas HSA is electronegative. The mean coverage $N$ of MTO on HSA was $12.4 \pm 1.02$, and the mean binding constant $K_{\mathrm{A}}$ was $(77.9 \pm 5.37) \times 10^{5} \mathrm{M}^{-1}$ (Table 1 ), so MTO had high affinity with HSA. With the MTO dropped, the reactions released or absorbed heat, but the whole reaction was an endothermic process (Figure 3(a)).
This finding may be due to the molar concentration of HSA being much less than that of MTO, which is diluted when the MTO is dropped, and the dilution process releases heat for hydration. The amount of heat release during each titration did not change, so the adsorption combination was independent of the dilution exotherm, and the reaction was mainly endothermic.

Figure 3(b) shows the HSA titration into CHP NPs. During the titration process, the alternate lines show the positive and negative peaks, and the total area of the positive peaks was definitely larger than the total area of the negative peaks. The value of the negative peaks was basically unchanged at each titration, maintained at about $-0.0125 \mu \mathrm{cal} / \mathrm{sec}$; the value of the positive peak decreased with increasing drops. The curve shows a downward trend, and the dots were scattered but steady and close to the curve. $\Delta H^{\circ}>0$ and $\Delta S^{\circ}>0$ indicate that the interaction between HSA and CHP NPs was mainly caused by hydrophobic interactions [33, 35]. CHP contains an inner hydrophobic core and an outer shell of hydrophilic groups, whereas HSA has hydrophobic amino acid residues. Therefore, CHP and HSA are close and bind with each other because of the hydrophobic interaction. According to $\triangle G^{\circ}=\triangle H^{\circ}-T \triangle S^{\circ}, \triangle G^{\circ}<0$ indicates that the adsorption reaction of HSA and CHP NPs proceeded spontaneously by the hydrophobic interaction. The mean coverage of HSA on CHP NPs was $1.17 \pm 0.044$, and the mean $K_{\mathrm{A}}$ was $(27.7 \pm 3.26) \times 10^{4} \mathrm{M}^{-1}$ (Table 1$)$, so the affinity between the two was high. The curve had an endothermic and exothermic part, but the latter was definitely larger than 
Time (min)

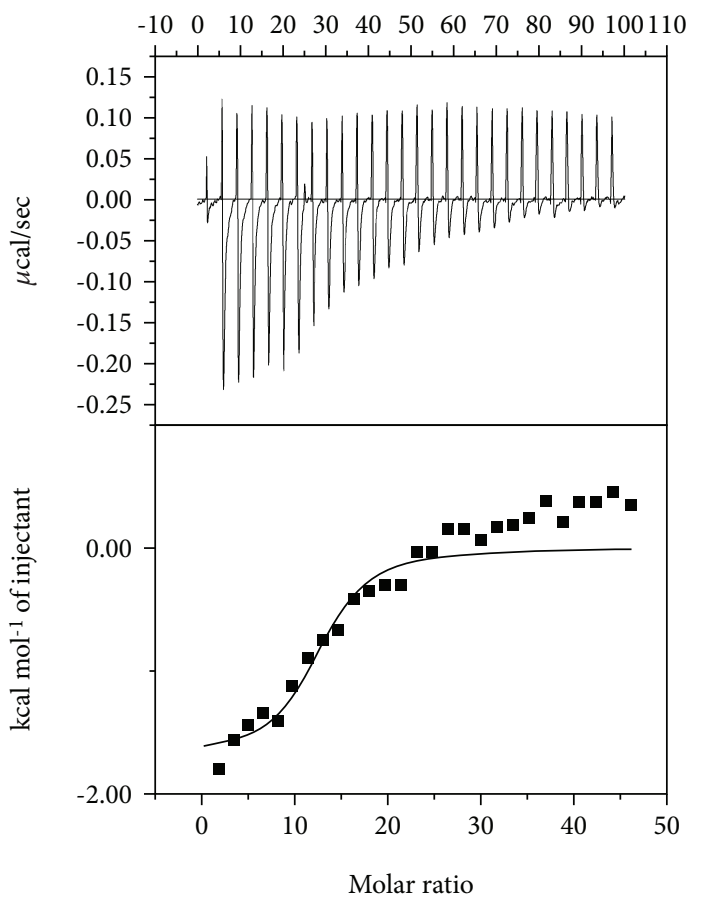

(a)
Time (min)

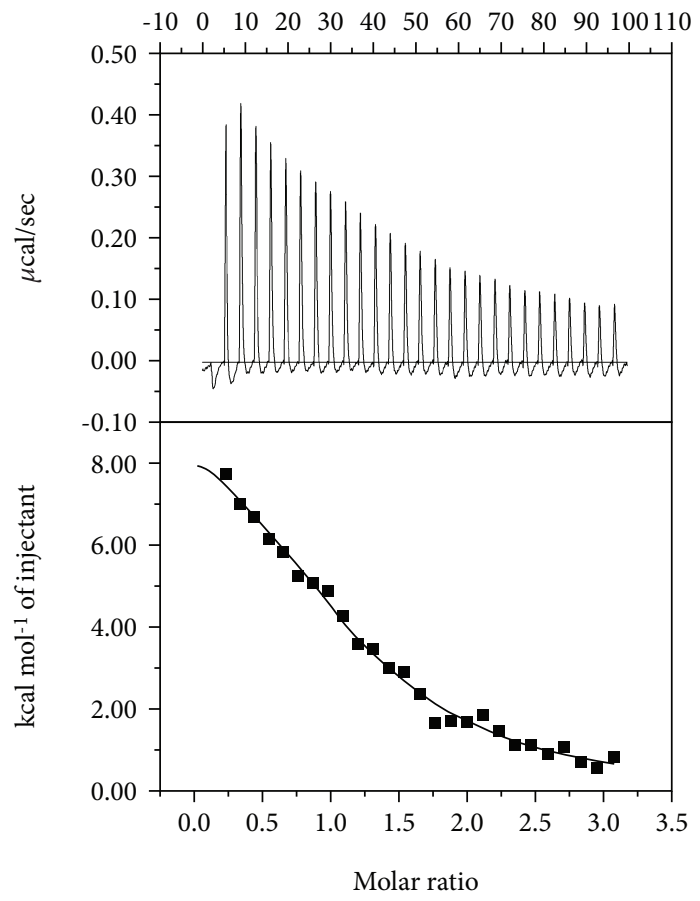

(b)

Time (min)

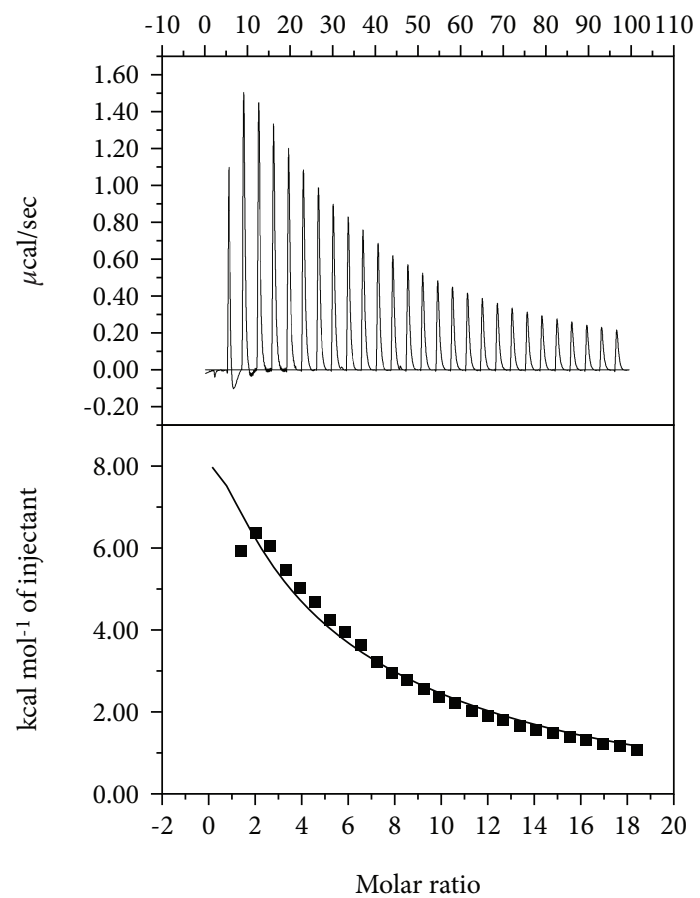

(c)

FIGURE 3: Isothermal calorimetry data for HSA titration into (a) MTO, (b) CHP, and (c) MTO-CHP NPs at $25^{\circ} \mathrm{C}$.

the former, finally showing an exothermic appearance. The endothermic part is likely due to the weak electronegativity of CHP NPs, which exerts the repulsive force of the same charge with HSA helping the system absorb minute amounts of heat during the HSA titration each time.
Figure 3(c) shows the HSA titrating into MTO-CHP NPs. The graph shows two small negative peaks at the first two drops in the experiment, which did not appear after that. A positive peak appeared after the first drop, and its value decreased with increasing drops. The corresponding curve 
TABle 1: Degree of coverage, affinity (binding constant, $K_{\mathrm{A}}$ ), and enthalpy and entropy changes for binding with HSA titrated into the following sample solutions.

\begin{tabular}{lcccc}
\hline Sample & Coverage & $K_{\mathrm{A}}\left(10^{4} \mathrm{M}^{-1}\right)$ & $\Delta H^{\circ}(\mathrm{kJ} / \mathrm{mol})$ & $\Delta S^{\circ}(\mathrm{kJ} / \mathrm{mol} \cdot \mathrm{K})$ \\
\hline MTO & $12.4 \pm 1.02$ & $77.9 \pm 5.37$ & $7.112 \pm 0.793$ & 0.089 \\
CHP & $1.17 \pm 0.044$ & $27.7 \pm 3.26$ & $4.387 \pm 2.353$ & 0.251 \\
MTO-CHP & $0.939 \pm 0.302$ & $0.832 \pm 0.278$ & $46.505 \pm 16.1$ & 1.634 \\
\hline
\end{tabular}

Data are mean \pm SD.

had a downward trend, and the dots were neat, basically falling on the curve. On the basis of $\Delta H^{\circ}>0, \triangle S^{\circ}>0$, to $\triangle G^{\circ}<0$, the reaction proceeded spontaneously, and the main driving force in the reaction was hydrophobic interaction between the two. The mean coverage of HSA on drug-loaded NPs was $0.939 \pm 0.302$, and the mean $K_{\mathrm{A}}$ was $(8.32 \pm 2.78) \times 10^{3} \mathrm{M}^{-1} \quad$ (Table 1$)$, so the affinity between the two was low. The curve does not show an endothermic phenomenon except for the first two drops, showing an exothermic phenomenon, because HSA takes the surface amino acid residues as active sites, adsorbs on the surface of MTO-CHP NPs by the hydrophobic reaction, and then gradually complexes into its hydrogel matrix. A part of the heat release should be considered to result from the emptying effect [35]. Hence, the entropy of the system was increased, and the free energy in the corresponding unit contact area was reduced. The endothermic part in the curve is likely due to the combination between the HSA molecule and the free MTO released from the MTO-CHP. Because the free MTO is only a small portion and the HSA increase goes faster than the drug release while the HSA is continuously titrated, gradually, the exothermic combinations of HSA and CHP NPs are predominant.

Both the coverage and binding constants were greater in Figure 3(a) than in Figure 3(b), so the affinity of HSA for MTO was higher than that of HSA for CHP NPs likely because of the electropositivity of MTO, electronegativity of HSA, and weak electronegativity of CHP NPs. HSA is easier to combine with oppositely charged species, so the affinity with MTO was higher.

Figures 3(a) and 3(c) show that the two binding effects were endothermic and exothermic, respectively. The binding constant and coverage were much higher in Figure 3(a) than in Figure 3(c), so the affinity of HSA was much higher for MTO than for MTO-CHP NPs. This finding may be due to MTO being encapsulated in CHP NPs, and the affinity of CHP NPs being much lower for HSA than MTO, resulting in decreased affinity for MTO-CHP NPs.

In contrast, Figures 3(b) and 3(c) both show exothermic phenomena. However, the binding constants and coverage were definitely less in Figure 3(c) than in Figure 3(b), reflecting the lower affinity of HSA for MTO-CHP NPs than CHP. This finding may be due to the competition between MTO and HSA in combination with CHP. MTO occupies the binding site of HSA on CHP NPs, and the binding of the two is restricted by each other, for decreased affinity of HSA for MTO-CHP NPs.
CHP NPs, as carrier-loaded NPs with MTO, could effectively solve the problem of drug clearance and the therapeutic effect caused by HSA binding, therefore exerting a better therapeutic effect. MTO-CHP NPs combined with HSA will result in slow release of MTO and prolonged duration of activity perhaps because of adsorption between MTO and HSA. HSA combined with CHP NPs and MTO in the same process could reduce the clearance rate of MTO.

3.4. Fluorescence Spectroscopy Determination of Interactions of Drug, CHP, and Drug-Loaded CHP NPs with HSA. The peak position and peak shape of the fluorescence emission peaks for HSA, HSA and MTO, HSA and CHP NPs, and HSA and MTO-CHP NPs were basically almost similar (Figures 4(a) and 4(b)). The maximum value of fluorescence emission intensities was at $342 \mathrm{~nm}$ owing to the effect of the tryptophan residue in the HSA molecule [27], so CHP NPs and HSA formed a complex. When the NPs were completely combined with HSA and at the beginning of the process, the fluorescence intensity for HSA was greatly decreased, and MTO-CHP NPs more strongly quenched HSA fluorescence intensity than did CHP NPs.

The maximum fluorescence intensity of HSA varied with complexation time (Figure 4(c)). When MTO was combined with HSA, the fluorescence intensity decreased within $3 \mathrm{~h}$, then remained stable, so the complexation of MTO and HSA completed quickly. When HSA was combined with $\mathrm{CHP}$ or MTO-CHP, the fluorescence intensity decreased rapidly within $0-6 \mathrm{~h}$ and slowly within $6-18 \mathrm{~h}$. The initially rapid decrease in fluorescence intensity was due to the fast adsorption of HSA to NPs, and the slow decrease was due to the gradual complexation between them. Therefore, we considered the compound obtained at $15 \mathrm{~h}$ as a completely formed complex.

3.5. Characterization of the Compound of HSA with DrugLoaded CHP NPs. The mean size distribution, PDI, and zeta potential changes for MTO-CHP NPs with HSA at $0,3,6,9$, 12, 15, and $18 \mathrm{~h}$ are in Figures 5(a) and 5(b) and Table 2. From 0 to $12 \mathrm{~h}$, the mean particle size increased from 89.91 to $144.4 \mathrm{~nm}$, which indicates the interaction between HSA and MTO-CHP NPs and the integration of HSA. From 12 to $18 \mathrm{~h}$, the increase in mean particle size slowed, so HSA was basically complexed with MTO-CHP NPs. PDI changed from 0.508 and 0.514 at the beginning of the reaction to 0.210 after $15 \mathrm{~h}$ of reaction, because multiple HSA was rapidly adsorbed on the surface of MTO-CHP NPs and there were many substances in the composite system. When HSA 


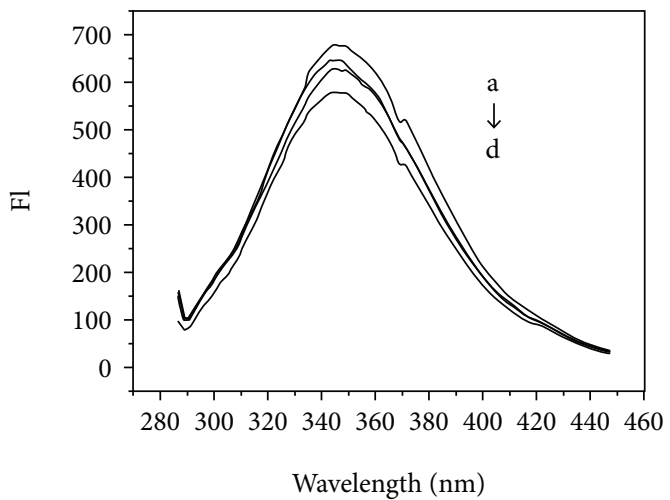

(a)

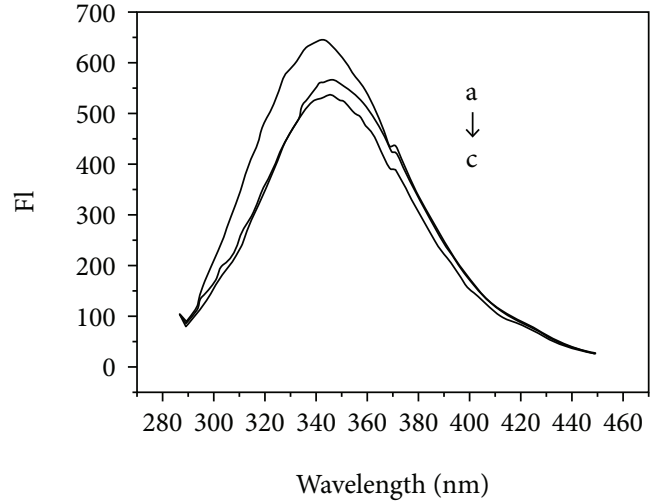

(b)

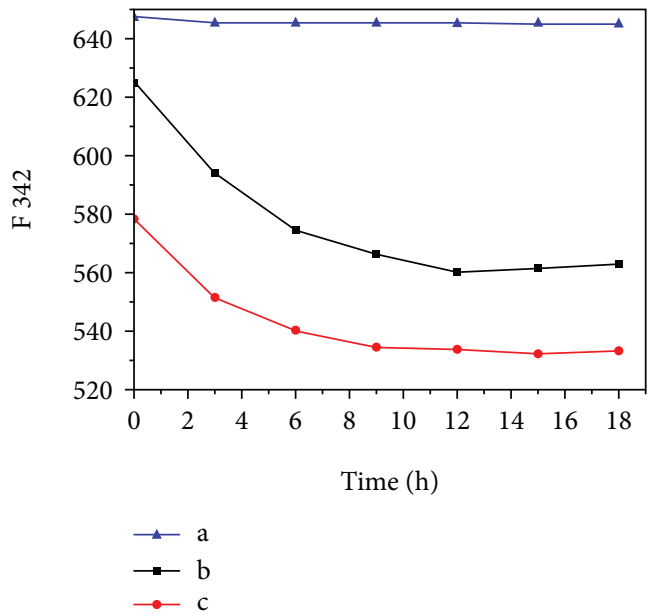

(c)

Figure 4: Fluorescence spectra for HSA in phosphate buffer with pH 7.40 at $25^{\circ} \mathrm{C}$. (a) a $\rightarrow \mathrm{d}$ represents the fluorescence spectra of the beginning of reaction for HSA, HSA and MTO, HSA and CHP NPs, and HSA and MTO-CHP NPs, respectively. (b) a $\rightarrow c$ represents the fluorescence spectra for HSA and MTO, HSA and CHP NPs, and HSA and MTO-CHP NPs, respectively, after the reaction was completed at $15 \mathrm{~h}$. (c) Change in maximum fluorescence intensity as a function of time combined with MTO (a), CHP NPs (b), and MTO-CHP NPs (c).

adsorbed on the surface gradually merged into the core of the NPs, the composite reaction was completed, and the dispersion was gradually stabilized with the decreased PDI value.

The zeta potential gradually changed from -15 to $-0.307 \mathrm{mV}$, because the charge of HSA was $-15 \mathrm{mV}$ [36], whereas MTO-CHP NPs were almost uncharged, which further indicated a composite reaction between HSA and MTO-CHP NPs. The charge was redistributed by electrostatic interaction, and the absolute value of the overall potential decreased.

TEM revealed that MTO-CHP NPs remained spherical after $15 \mathrm{~h}$ of reaction with HSA (Figure 5(c)). HSA did not change the morphology of MTO-CHP NPs. HSA may enter into the core of MTO-CHP NPs or be evenly distributed on the surface of MTO-CHP NPs.

3.6. Changes in Secondary Structure with Drug-Loaded CHP NPs Combined with HSA. The CD spectra for HSA shows a negative peak at $208 \mathrm{~nm}$, considered as a characteristic peak of the $\alpha$-helical structure [37]. The proportion of $\alpha$-helix contents for free HSA was $52.3 \%$, and that at the beginning of the adsorption of MTO, CHP, and MTO-CHP decreased to $50.0 \%, 46.9 \%$, and $43.7 \%$, respectively (Table 3 ). The proportion after maximum adsorption of MTO, CHP, and MTO-CHP was $49.4 \%, 41.8 \%$, and $39.1 \%$, respectively (Figures 6(a) and 6(b)). The ellipticity at $208 \mathrm{~nm}$ was almost unchanged after $3 \mathrm{~h}$ when HSA interacted with MTO but was stabilized until $9 \mathrm{~h}$ when HSA interacted with CHP and MTO-CHP (Figure 6(c)). Thus, the HSA adsorption of MTO was rapid; moreover, MTO changed the secondary structure of HSA to a small extent. The combination of HSA with CHP and MTO-CHP was slow, and HSA complexed into its hydrogel matrix gradually. During this process, HSA was pulled by the hydrophobic interaction of the CHP hydrophobic core and the hydrophilic polysaccharide chain of the CHP surface, causing the HSA to deform and altering its secondary structure. Because of the double complex reaction between MTO-CHP and HSA (i.e., the interaction between CHP and HSA, MTO, and HSA), the secondary structure of HSA changed to a greater extent. 


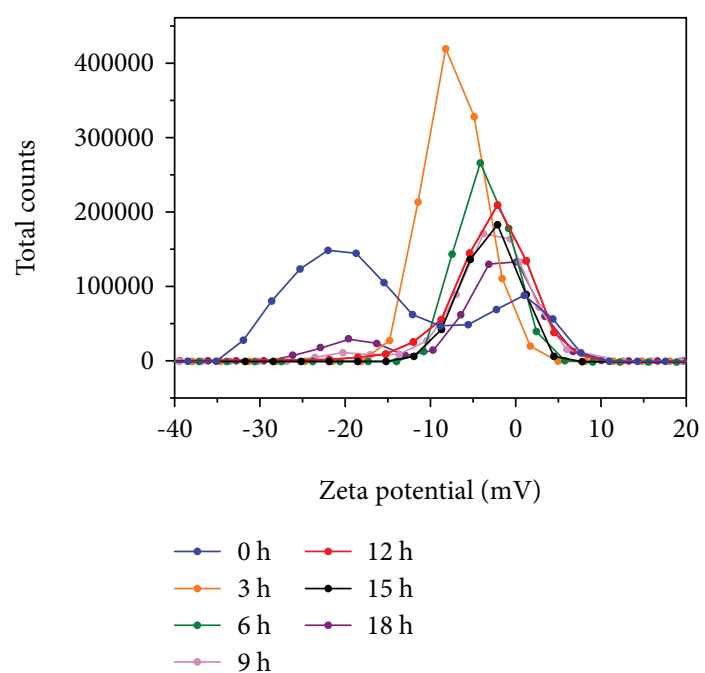

(a)

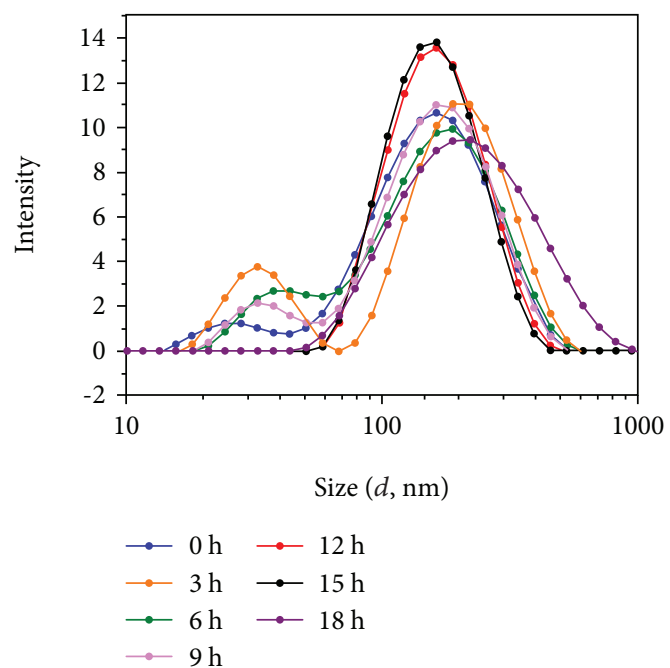

(b)

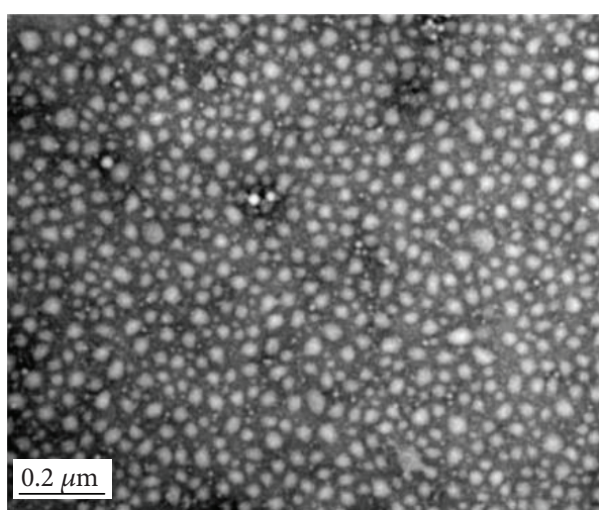

(c)

Figure 5: Zeta potential (a), size distribution (b), and transmission electron microscopy (TEM) images (c) of HSA-MTO-CHP NPs, with different times of complexation.

TABLE 2: Mean size distribution, polydispersity index (PDI), and zeta potential changes of MTO-CHP NPs and HSA at different combination times.

\begin{tabular}{lccccccc}
\hline & \multicolumn{7}{c}{ Time (h) } \\
& 0 & 3 & 6 & 9 & 12 & 15 & 18 \\
\hline Size $(\mathrm{nm})$ & 89.91 & 109.4 & 114.3 & 120.4 & 144.4 & 144.7 & 145.2 \\
PDI & 0.508 & 0.514 & 0.304 & 0.285 & 0.199 & 0.210 & 0.231 \\
Zeta $(\mathrm{mV})$ & -15 & -7.22 & -4.7 & -3.74 & -3.46 & -3.08 & -3.073 \\
\hline
\end{tabular}

TABLE 3: The $\alpha$-helix contents of HSA, including free HSA (Aa), HSA in the presence of MTO (Ab), CHP NPs (Ac), MTO-CHP NPs (Ad), and HSA completely combined with MTO (Ba), CHP NPs (Bb), and MTO-CHP NPs (Bc).

\begin{tabular}{lccccccc}
\hline Project & $\mathrm{Aa}$ & $\mathrm{Ab}$ & $\mathrm{Ac}$ & $\mathrm{Ad}$ & $\mathrm{Ba}$ & $\mathrm{Bb}$ & $\mathrm{Bc}$ \\
\hline$\alpha$-Helix (\%) & 52.3 & 50.0 & 46.9 & 43.7 & 49.4 & 41.8 & 39.1 \\
\hline
\end{tabular}

3.7. Sustained Drug Release after HSA Complexed with Drug-Loaded CHP NPs. Figure 7 shows the MTO release profile of MTO-CHP NPs in media with $\mathrm{pH} 5.6$ or 7.4 and a composite with HSA in media with $\mathrm{pH} 5.6$ or 7.4. MTO release had a biphasic character; that is, the drug was released rapidly in the first $12 \mathrm{~h}$, at $56.73 \%, 39.82 \%$, $32.45 \%$, and $28.02 \%$, respectively, and the drug release rate was slowed down within 12 to $48 \mathrm{~h}$, with cumulative release percentages of $68.75 \%, 50.54 \%, 35.22 \%$, and $31.68 \%$, respectively. Because NPs capture hydrophobic drugs in two forms of loading, surface adsorption, and core loading, the surfaceadsorbed drug was rapidly released, whereas the core-loaded drug was slowly and continuously released [38]. The drug release of the NPs complexed with HSA was much slower. This finding might have two explanations. First, HSA is rapidly bound to the surface of CHP NPs and then slowly enters the NP core and binds to the CHP hydrophobic domain, forming steric hindrance and weakening the hydrophobic interaction between MTO and CHP. Second, during the MTO release process, it may combine with the HSA adsorbed by the NPs. The drug release of MTO-CHP NPs was faster at $\mathrm{pH} 5.6$ than at $\mathrm{pH} 7.4$, mainly because the degree of protonation of MTO and water solubility became larger under 


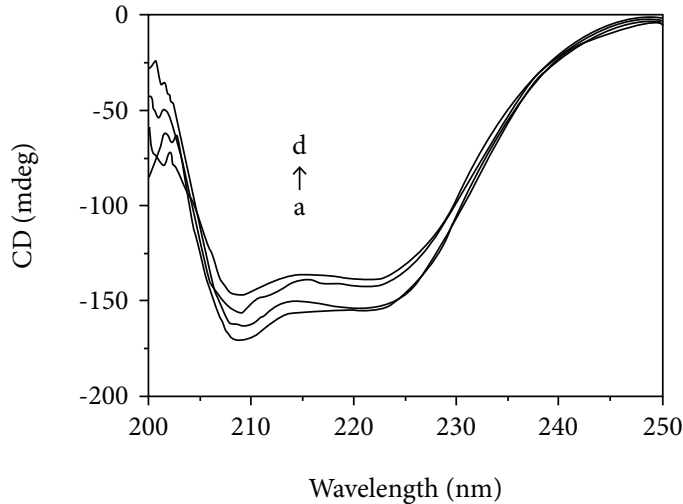

(a)

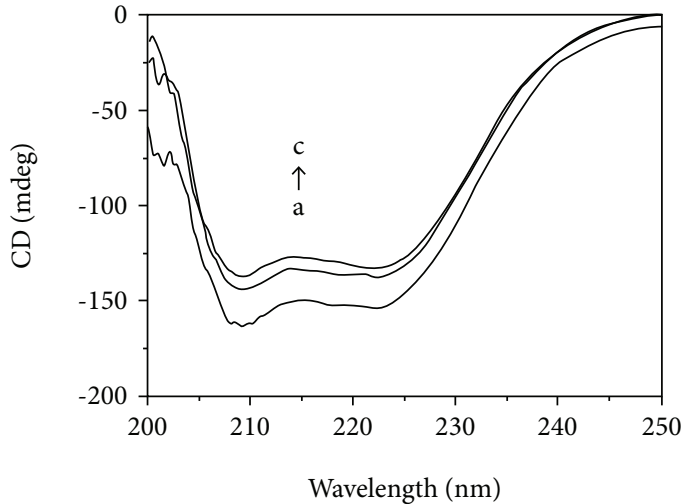

(b)

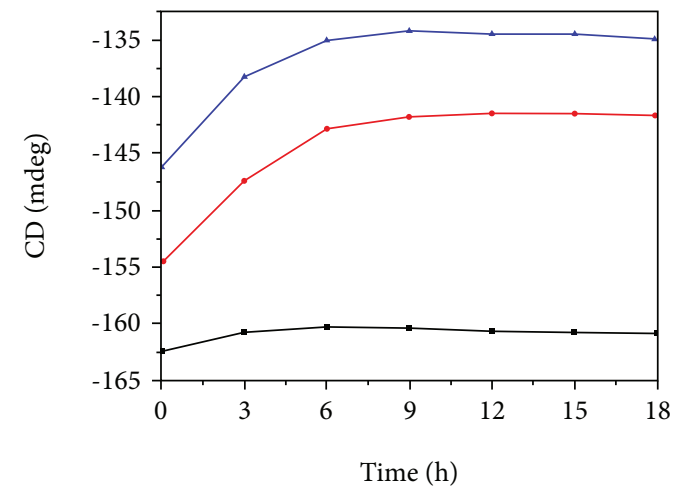

$\rightarrow$ HSA-MTO-CHP NPs
$\rightarrow$ HSA-CHP NPs
$\rightarrow$ HSA-MTO

(c)

Figure 6: Far-ultraviolet circular dichroism of HSA determined at $\mathrm{pH} 7.4$ Tris- $\mathrm{HCl}$ buffer and constant temperature $25^{\circ} \mathrm{C}$. (a) a $\rightarrow \mathrm{d}$ represents CD changes of HSA (Aa), HSA and MTO (Ab), HSA and CHP NPs (Ac), and HSA and MTO-CHP NPs (Ad) at the beginning of complexation. (b) $\mathrm{a} \rightarrow \mathrm{c}$ represents the circular dichroism of HSA and MTO (Ba), HSA and CHP NPs (Bb), and HSA and MTO-CHP NPs $(\mathrm{Bc})$ at $15 \mathrm{~h}$ of complexation. (c) The ellipticity of HSA at $208 \mathrm{~nm}$ corresponding to the reaction time with various substances.

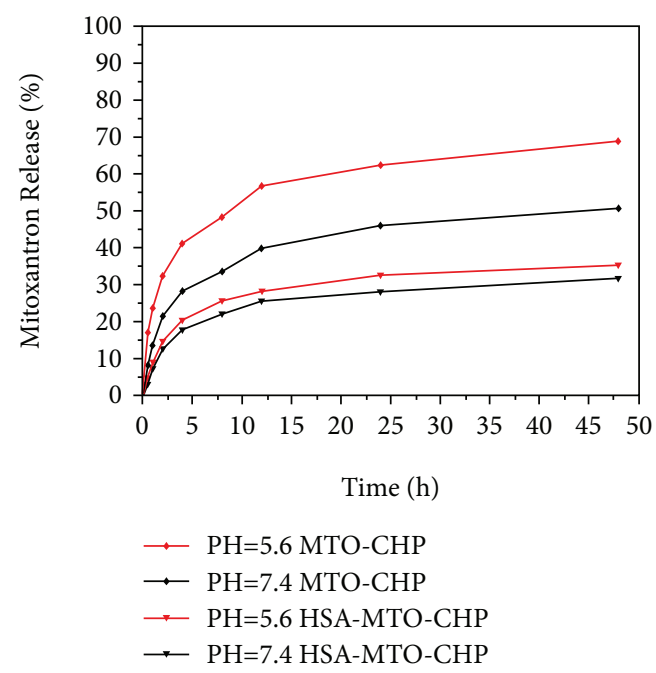

FIGURE 7: MTO release curve of different NPs in media with $\mathrm{pH} 5.6$ $(\diamond, \mathrm{MTO}-\mathrm{CHP} ; \boldsymbol{\nabla}, \mathrm{HSA}-\mathrm{MTO}-\mathrm{CHP})$ and $7.4(\diamond, \mathrm{MTO}-\mathrm{CHP} ; \boldsymbol{\nabla}$, HSA-MTO-CHP). acidic conditions [39]. However, the MTO-CHP NPs complexed with HSA had a low drug release rate under both pH 7.4 (physiological condition) and pH 5.6 (acidic condition) because the steric hindrance of HSA formation is large, and this property is not affected by acidity or alkalinity.

\section{Discussion}

HSA can react with small molecular drugs, NPs, and drugloaded NPs [40-42]. However, a number of fundamental principles have not been elucidated, including the binding force, binding coefficient, type of reaction, relationship between active and passive adsorption, and characteristics of their interactions [43-45]. The combination of HSA with drugs, NPs, and drug-loaded NPs definitely affects their structure, distribution, toxicity, and effectiveness of related drugs [46]. Understanding the characteristics of HSAassociated interactions, especially the HSA effect on drug release from drug-loaded NPs, holds great potential for improving drug delivery in the clinical applications.

We prepared CHP NPs loaded with MTO (MTO-CHP NPs) with size $166.9 \mathrm{~nm}$. The interaction between 
MTO-CHP NPs was mainly by hydrophobic interaction force, electrostatic force, and hydrogen bond. MTO-CHP NPs could quench the fluorescence intensity of HSA. And the fluorescence intensity gradually decreased to be balanced at $9 \mathrm{~h}$. At the same time, the particle size and potential remained stable, accompanied by a size change from 89.91 to about $145 \mathrm{~nm}$ and potential change from -15 to $-3 \mathrm{mV}$. With MTO-CHP NPs combined with HSA, the change in ellipticity of HSA at $208 \mathrm{~nm}$ was similar to that of fluorescence spectra and DLS measurements. The proportion of $\alpha$-helixes was decreased until combination for $9 \mathrm{~h}$. The release of MTO from MTO-CHP NPs at $\mathrm{pH}=5.6$ was significantly accelerated, whereas that of MTO from HSA-MTO-CHP NPs was significantly reduced. And the drug release was significantly slowed down even under acidic conditions, which indicates the beneficial effect of HSA on the persistence and stability of the HSA-MTO-CHP NP compound.

In general, the interaction of HSA with small molecular drugs, such as naproxen, ibuprofen, and flurbiprofen, is relatively simple, rapid, and reversible, reaching a saturation state in a short time [47]. Two mechanisms are believed involved in the interaction between NPs and HSA. The first is the "nanocrystalline corona," whereby small NPs produce a corona on the surface of the protein [48]. The second is the "protein corona," whereby proteins form a corona around NPs. The combination of different NPs cannot be separated from electrostatic interaction, van der Waals force, hydrogen bond, and hydrophobic interaction [49].

The reaction between drug-loaded NPs and HSA is complicated. Some drugs form strong space resistance and block the reaction of HSA with drug-loaded NPs. For example, after pHPMA NPs are loaded with DOX, the structure cannot be captured by the network structure formed by the pHPMA chain or diffused through the loose pHPMA NP shell into the cholesterol core of pHPMA NPs [15]. Ghosh et al. studied the interaction of MPNPs and HSA with morin. Morin in the MPNP surface initially bound to HSA, forming morin-HSA, which in turn bound to the NP surface. Morin encapsulated in the MPNPs matrix may also be adsorbed by the complexed HSA, resulting in a surface-induced steric resistance effect, which limited the release of morin from MPNPs [16].

In the present study, we systematically investigated the reaction of MTO-CHP NPs with HSA. As shown in Figure 2, HSA interacted with MTO-CHP NPs similar to CHP NPs and HSA. Because of the hydrophobic interaction, HSA was rapidly adsorbed to the surface of MTO-CHP NPs by electrostatic interaction. Because of the hydrophobic section of the NP core and the resistance of the hydrophilic polysaccharide chain on its surface, HSA gradually complexed into the core of NPs and combined with the cholesterol group, forming HSA-MTO-CHP NPs. In DOX-loaded pHPMA NPs, the hydrophobicity of DOX is smaller than that of MTO, and DOX is distributed in the PHPMA shell, which results in large steric resistance hindering the adsorption of HSA [15]. However, the mean coverage of HSA and MTO-CHP NPs was $0.939 \pm 0.302$, and the mean binding constant was $0.832 \pm 0.278 \times 10^{4} \mathrm{M}^{-1}$, which was much smaller than that for HSA and CHP NPs. The fluorescence quenching of HSA was evident, accompanied by a significant decrease in $\alpha$-helix content in HSA.

According to previous research [22], MTO can react with HSA and stabilize domain I in the hydrophobic region of HSA via hydrophobic interaction, hydrogen bonding, and electrostatic interaction. The CHP NPs package MTO prepared by dialysis self-assembly, physical adsorption, or MTO-coated CHP NPs, and the latter is encapsulated in the hydrophobic core of CHP NPs via hydrophobic interaction. So, the interaction between HSA and MTO-CHP NPs may differ from that of HSA and CHP NPs for two reasons. First, MTO reacts with HSA. When the combined drug is released, drug release is hindered by the presence of HSA. In addition, the high affinity between MTO and HSA indicates that MTO is secondarily combined with HSA adsorbed on CHP NPs. This result is consistent with Ghosh et al. [16]. Second, HSA competes with MTO. Both HSA and MTO bind to the hydrophobic region of CHP NPs via hydrophobic interaction. Our ITC results showed that the affinity and coverage of HSA and MTO-CHP NPs were relatively low. All features met our expectations.

Blood contains opsonins, integrins, immunoglobulins, complement proteins, fibrinogens, etc., which are easily adsorbed on the surface of NPs, thereby promoting the recognition and phagocytosis of NPs by macrophages and removing NPs from the blood or their accumulation in immune organs such as liver, spleen, and bone marrow. The targeting of drug-loaded nanometers is interfered by combination with these proteins $[50,51]$. However, HSA-complexed NPs could block the absorption of other serum proteins including opsonin and integrin [52], thereby prolonging the blood circulation of drug-loaded NPs in vivo and enhancing their passive targeting.

The design and application of nanoformulations require understanding the interaction between drug-loaded NPs and HSA. HSA-complexed NPs can be used as an ideal model to explore the potential applications of albumin nanosystems in vivo.

\section{Conclusion}

This study found that MTO-CHP NPs interact with HSA, with hydrophobic interactions playing a major role. Combining HSA and MTO-CHP NPs is a slow process. Because of the hydrophobic interaction, HAS first adsorbs on the surface of CHP-MTO NPs, then gradually complexes into its hydrogel matrix, resulting in the increased size and potential changes of MTO-CHP NPs. In addition, the combination alters the secondary structure of HSA and its $\alpha$-helix content. In addition, the complexation of HSA enhances the sustained MTO release from MTO-CHP NPs, and the drug release adapts to acidic conditions, thereby improving the stability of the drug release.

\section{Abbreviations}

CHP:

MTO:

NPs:

Hydrophobically modified pullulan Mitoxantrone

Nanoparticles 


$\begin{array}{ll}\text { HSA: } & \text { Human serum albumin } \\ \text { MTO-CHP NPs: } & \begin{array}{l}\text { Mitoxantrone-loaded hydrophobi- } \\ \text { cally modified pullulan NPs }\end{array} \\ \text { HSA-MTO-CHP NPs: } & \text { HSA combined with MTO-CHP } \\ & \text { NPs. }\end{array}$

\section{Data Availability}

The data used to support the findings of this study are available from the corresponding author upon request.

\section{Conflicts of Interest}

The authors declare that they have no conflicts of interest.

\section{Authors' Contributions}

Liming Yuan and Bu Guo contributed equally to this work.

\section{Acknowledgments}

This project was supported by the Health and Family Planning Commission of Hunan Province research project (no. B2017073) to Xiaojun Tao, National Natural Science Foundation of China (nos. 81303254 and 81641140) and Hubei Province Health and Family Planning Scientific Research Project (no. WJ2017M214) to Qiufang Zhang, and the Natural Science Foundation of Hunan Province (no. 2016JJ2088) to Liming Yuan.

\section{References}

[1] J. H. Nam, S. Y. Kim, and H. Seong, "Investigation on physicochemical characteristics of a nanoliposome-based system for dual drug delivery," Nanoscale Research Letters, vol. 13, no. 1, pp. 101-112, 2018.

[2] G. Yan, A. Li, A. Zhang, Y. Sun, and J. Liu, "Polymer-based nanocarriers for co-delivery and combination of diverse therapies against cancers," Nanomaterials, vol. 8, no. 2, pp. 85-112, 2018.

[3] J. Shi, P. W. Kantoff, R. Wooster, and O. C. Farokhzad, "Cancer nanomedicine: progress, challenges and opportunities," Nature Reviews. Cancer, vol. 17, no. 1, pp. 20-37, 2017.

[4] U. Roy, H. Ding, S. Pilakka Kanthikeel et al., "Preparation and characterization of anti-HIV nanodrug targeted to microfold cell of gut-associated lymphoid tissue," International Journal of Nanomedicine, vol. 10, no. 1, pp. 5819-5835, 2015.

[5] M. P. Calatayud, B. Sanz, V. Raffa, C. Riggio, M. R. Ibarra, and G. F. Goya, "The effect of surface charge of functionalized Fe3O4 nanoparticles on protein adsorption and cell uptake," Biomaterials, vol. 35, no. 24, pp. 6389-6399, 2014.

[6] J. Giri, M. S. Diallo, A. J. Simpson et al., "Interactions of poly(amidoamine) dendrimers with human serum albumin: binding constants and mechanisms," ACS Nano, vol. 5, no. 5, pp. 3456-3468, 2011.

[7] X. Tao, S. Jin, D. Wu et al., "Effects of particle hydrophobicity, surface charge, media $\mathrm{pH}$ value and complexation with human serum albumin on drug release behavior of mitoxantroneloaded pullulan nanoparticles," Nanomaterials (Basel), vol. 6, no. 1, p. $2,2016$.
[8] S. Zhang, Y. Moustafa, and Q. Huo, "Different interaction modes of biomolecules with citrate-capped gold nanoparticles," ACS Applied Materials \& Interfaces, vol. 6, no. 23, pp. 21184-21192, 2014.

[9] Y.-J. Hu, Y. Ou-Yang, C.-M. Dai, Y. Liu, and X.-H. Xiao, "Site-selective binding of human serum albumin by palmatine: spectroscopic approach," Biomacromolecules, vol. 11, no. 1, pp. 106-112, 2010.

[10] J. Ghuman, P. A. Zunszain, I. Petitpas, A. A. Bhattacharya, M. Otagiri, and S. Curry, "Structural basis of the drugbinding specificity of human serum albumin," Journal of Molecular Biology, vol. 353, no. 1, pp. 38-52, 2005.

[11] M. M. McCallum, A. J. Pawlak, W. R. Shadrick et al., "A fluorescence-based high throughput assay for the determination of small molecule-human serum albumin protein binding," Analytical and Bioanalytical Chemistry, vol. 406, no. 7, pp. 1867-1875, 2014.

[12] J. X. Fu, K. W. Wang, Y. S. Ge et al., "Biophysical studies of the interaction between a triazole derivative and bovine serum albumin by multi-spectroscopic and molecular modeling methods," Science China Chemistry, vol. 54, no. 5, pp. 788796, 2011.

[13] S. Huang, H. Qiu, J. Xie et al., "Systematical investigation of in vitro molecular interaction between fluorescent carbon dots and human serum albumin," RSC Advances, vol. 6, no. 50, pp. 44531-44542, 2016.

[14] C. Ruan, L. Liu, Y. Lu et al., "Substance P-modified human serum albumin nanoparticles loaded with paclitaxel for targeted therapy of glioma," Acta Pharmaceutica Sinica B, vol. 8, no. 1, pp. 85-96, 2018.

[15] X. Zhang, B. J. Niebuur, P. Chytil et al., "Macromolecular pHPMA-based nanoparticles with cholesterol for solid tumor targeting: behavior in HSA protein environment," Biomacromolecules, vol. 19, no. 2, pp. 470-480, 2018.

[16] P. Ghosh, J. Patwari, and S. Dasgupta, "Complexation with human serum albumin facilitates sustained release of morin from polylactic-co-glycolic acid nanoparticles," Journal of Physical Chemistry B, vol. 121, no. 8, pp. 1758-1770, 2017.

[17] M. Szwed, "Mitoxantrone - an anthraquinone antibiotic with antitumor activity applied for the treatment of multiple sclerosis," Postępy Higieny i Medycyny Doświadczalnej (Online), vol. 68, pp. 198-208, 2014.

[18] K. Akiyoshi, T. Nishikawa, Y. Mitsui, T. Miyata, M. Kodama, and J. Sunamoto, "Self-assembly of polymer amphiphiles: thermodynamics of complexation between bovine serum albumin and self-aggregate of cholesterol-bearing pullulan," Colloids and Surfaces A: Physicochemical and Engineering Aspects, vol. 112, no. 2-3, pp. 91-95, 1996.

[19] R. S. Singh, N. Kaur, and J. F. Kennedy, "Pullulan and pullulan derivatives as promising biomolecules for drug and gene targeting," Carbohydrate Polymers, vol. 123, pp. 190-207, 2015.

[20] H. Li, S. Bian, Y. Huang, J. Liang, Y. Fan, and X. Zhang, "High drug loading $\mathrm{pH}$-sensitive pullulan-DOX conjugate nanoparticles for hepatic targeting," Journal of Biomedical Materials Research Part A, vol. 102, no. 1, pp. 150-159, 2014.

[21] M. G. Antoniraj, M. Ayyavu, L. J. K. Henry et al., "Cytocompatible chitosan-graft-mPEG-based 5-fluorouracil-loaded polymeric nanoparticles for tumor-targeted drug delivery," Drug Development and Industrial Pharmacy, vol. 44, no. 3, pp. 365-376, 2018. 
[22] S. N. Khan, B. Islam, R. Yennamalli, A. Sultan, N. Subbarao, and A. U. Khan, "Interaction of mitoxantrone with human serum albumin: spectroscopic and molecular modeling studies," European Journal of Pharmaceutical Sciences, vol. 35, no. 5, pp. 371-382, 2008.

[23] X. Tao, T. Tao, Y. Wen et al., "Novel delivery of mitoxantrone with hydrophobically modified pullulan nanoparticles to inhibit bladder cancer cell and the effect of nano-drug size on inhibition efficiency," Nanoscale Research Letters, vol. 13, no. $1,2018$.

[24] W. Yang, H.-1. Chen, F.-p. Gao et al., "Self-aggregated nanoparticles of cholesterol-modified pullulan conjugate as a novel carrier of mitoxantronep," Current Nanoscience, vol. 6, no. 3, pp. 298-306, 2010.

[25] C. L. Zhang, Y. X. Liu, X. M. Zhang et al., "Synthesis, characterization, DNA/HSA interactions and in vitro cytotoxic activities of two novel water-soluble copper(II) complexes with 1,3,5-triazine derivative ligand and amino acids," Materials Science and Engineering: C, vol. 91, pp. 414-425, 2018.

[26] S. Beigoli, A. Sharifi Rad, A. Askari, R. Assaran Darban, and J. Chamani, "Isothermal titration calorimetry and stopped flow circular dichroism investigations of the interaction between lomefloxacin and human serum albumin in the presence of amino acids," Journal of Biomolecular Structure \& Dynamics, vol. 37, no. 9, pp. 2265-2282, 2018.

[27] S. Cong, J. Bi, X. Song, C. Yu, and M. Tan, "Ultrasmall fluorescent nanoparticles derived from roast duck: their physicochemical characteristics and interaction with human serum albumin," Food and Function, vol. 9, no. 4, pp. 2490-2495, 2018.

[28] M. B. Bolattin, S. T. Nandibewoor, S. D. Joshi, S. R. Dixit, and S. A. Chimatadar, "Interaction of hydralazine with human serum albumin and effect of $\beta$-cyclodextrin on binding: insights from spectroscopic and molecular docking techniques," Industrial and Engineering Chemistry Research, vol. 55, no. 19, pp. 5454-5464, 2016.

[29] A. Micsonai, F. Wien, L. Kernya et al., "Accurate secondary structure prediction and fold recognition for circular dichroism spectroscopy," Proceedings of the National Academy of Sciences of the United States of America, vol. 112, no. 24, pp. E3095-E3103, 2015.

[30] N. Shahabadi, S. Hadidi, and Z. M. Kalar, "Biophysical studies on the interaction of platinum(II) complex containing antiviral drug ribavirin with human serum albumin," Journal of Photochemistry and Photobiology. B, vol. 160, pp. 376-382, 2016.

[31] A. Rabbani-Chadegani and Z. Hajihassan, "The effect of mitoxantrone as an anticancer drug on hepatocytes nuclei and chromatin: Selective release of histone proteins," Indian Journal of Pharmacology, vol. 43, no. 2, pp. 187-191, 2011.

[32] B. Hong and Q. Cheng, "Sensitive voltammetric determination of mitoxantrone by using CS-dispersed graphene modified glassy carbon electrodes," Advances in Chemical Engineering and Science, vol. 02, no. 04, pp. 453-460, 2012.

[33] P. D. Ross and S. Subramanian, "Thermodynamics of protein association reactions: forces contributing to stability," Biochemistry, vol. 20, no. 11, pp. 3096-3102, 1981.

[34] F. Biedermann, V. D. Uzunova, O. A. Scherman, W. M. Nau, and A. de Simone, "Release of high-energy water as an essential driving force for the high-affinity binding of cucurbit[n] urils," Journal of the American Chemical Society, vol. 134, no. 37, pp. 15318-15323, 2012.
[35] M. A. K. Azad, J. X. Huang, M. A. Cooper et al., "Structureactivity relationships for the binding of polymyxins with human $\alpha$-1-acid glycoprotein," Biochemical Pharmacology, vol. 84, no. 3, pp. 278-291, 2012.

[36] I. L. de Redín, C. B. María, C. Martínez-Ohárriz et al., "Human serum albumin nanoparticles for ocular delivery of bevacizumab," International Journal of Pharmaceutics, vol. 541, no. 12, pp. 214-223, 2018.

[37] Y. Wang, Q. Jiang, L. R. Liu, and Q. Zhang, “The interaction between bovine serum albumin and the self-aggregated nanoparticles of cholesterol-modified O-carboxymethyl chitosan," Polymer, vol. 48, no. 14, pp. 4135-4142, 2007.

[38] Y. Chen, D. Wu, W. Zhong et al., "Evaluation of the PEG density in the PEGylated chitosan nanoparticles as a drug carrier for curcumin and mitoxantrone," Nanomaterials, vol. 8, no. 7, pp. 486-4142, 2018.

[39] G. A. Ilevbare, H. Liu, K. J. Edgar, and L. S. Taylor, "Effect of binary additive combinations on solution crystal growth of the poorly water-soluble drug, ritonavir," Crystal Growth and Design, vol. 12, no. 12, pp. 6050-6060, 2012.

[40] H. Yang, J. Liu, Y. Huang et al., “Domain-specific interactions between MLN8237 and human serum albumin estimated by STD and WaterLOGSY NMR, ITC, spectroscopic, and docking techniques," Scientific Reports, vol. 7, no. 1, pp. 4551445525, 2017.

[41] D. Shcharbin, E. Pedziwiatr-Werbicka, T. Serchenya et al., "Role of cationic carbosilane dendrons and metallic core of functionalized gold nanoparticles in their interaction with human serum albumin," International Journal of Biological Macromolecules, vol. 118, no. Part B, pp. 1773-1780, 2018.

[42] D. M. Dhoke, S. S. Basaiyye, and P. B. Khedekar, "Development and characterization of L-HSA conjugated PLGA nanoparticle for hepatocyte targeted delivery of antiviral drug," Journal of Drug Delivery Science and Technology, vol. 47, pp. 77-94, 2018.

[43] D. Wu, J. Yan, J. Wang, Q. Wang, and H. Li, "Characterisation of interaction between food colourant allura red AC and human serum albumin: multispectroscopic analyses and docking simulations," Food Chemistry, vol. 170, pp. 423-429, 2015.

[44] C. Ràfols, S. Zarza, and E. Bosch, "Molecular interactions between some non-steroidal anti-inflammatory drugs (NSAID's) and bovine (BSA) or human (HSA) serum albumin estimated by means of isothermal titration calorimetry (ITC) and frontal analysis capillary electrophoresis (FA/CE)," Talanta, vol. 130, pp. 241-250, 2014.

[45] D. Sharma, M. Ashaduzzaman, M. Golabi, A. Shriwastav, K. Bisetty, and A. Tiwari, "Studies on bacterial proteins corona interaction with saponin imprinted $\mathrm{ZnO}$ nanohoneycombs and their toxic responses," ACS Applied Materials \& Interfaces, vol. 7, no. 43, pp. 23848-23856, 2015.

[46] D. OWENSIII and N. PEPPAS, "Opsonization, biodistribution, and pharmacokinetics of polymeric nanoparticles," International Journal of Pharmaceutics, vol. 307, no. 1, pp. 93-102, 2006.

[47] M. V. Zyuzin, Y. Yan, R. Hartmann et al., "Role of the protein corona derived from human plasma in cellular interactions between nanoporous human serum albumin particles and endothelial cells," Bioconjugate Chemistry, vol. 28, no. 8, pp. 2062-2068, 2017.

[48] V. H. Nguyen, N. M. Meghani, H. H. Amin et al., "Modulation of serum albumin protein corona for exploring cellular 
behaviors of fattigation-platform nanoparticles," Colloids and Surfaces B Biointerfaces, vol. 170, pp. 179-186, 2018.

[49] H. Rimac, Ž. Debeljak, M. Bojić, and L. Miller, "Displacement of drugs from human serum albumin: from molecular interactions to clinical significance," Current Medicinal Chemistry, vol. 24, no. 18, pp. 1930-1947, 2017.

[50] D. Wrobel, M. Marcinkowska, A. Janaszewska et al., "Influence of core and maltose surface modification of PEIs on their interaction with plasma proteins-human serum albumin and lysozyme," Colloids and Surfaces. B, Biointerfaces, vol. 152, pp. 18-28, 2017.

[51] X. Montet, K. Montet-Abou, F. Reynolds, R. Weissleder, and L. Josephson, "Nanoparticle imaging of integrins on tumor cells," Neoplasia, vol. 8, no. 3, pp. 214-222, 2006.

[52] S. N. dos Santos, S. R. Rezende Dos Reis, L. P. Pires et al., "Avoiding the mononuclear phagocyte system using human albumin for mesoporous silica nanoparticle system," Microporous and Mesoporous Materials, vol. 251, pp. 181-189, 2017. 


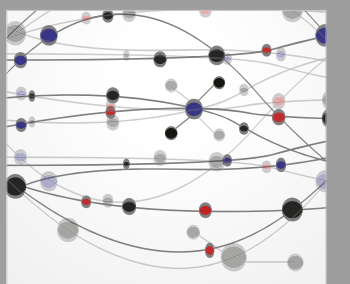

The Scientific World Journal
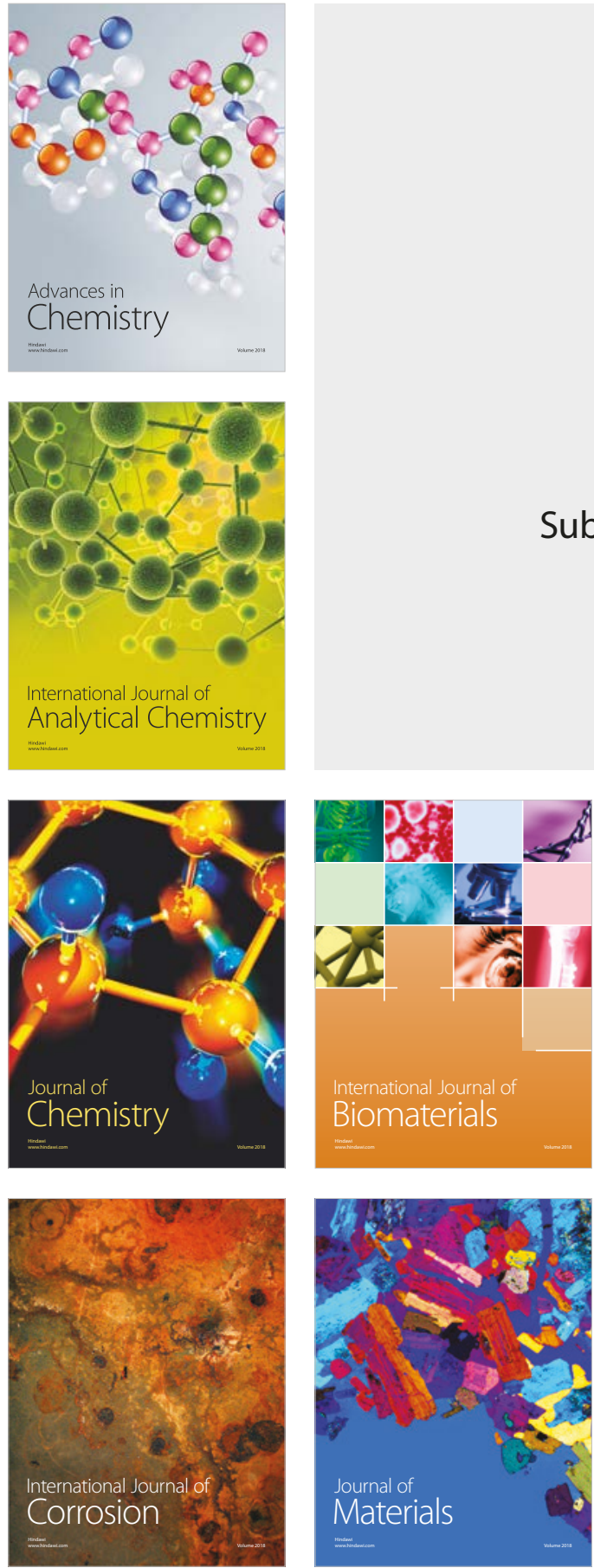

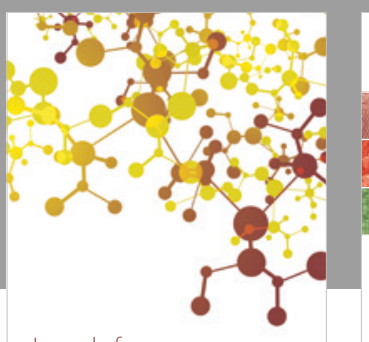

Journal of

Applied Chemistry
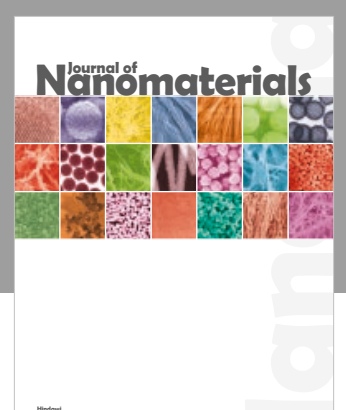

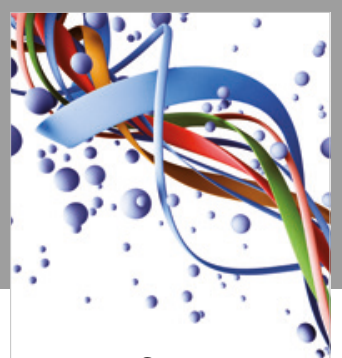

Scientifica

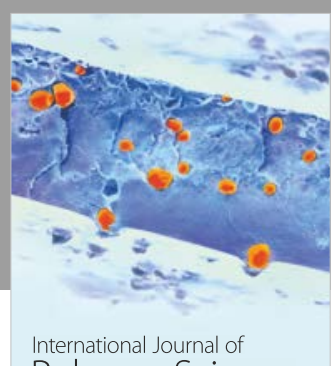

Polymer Science

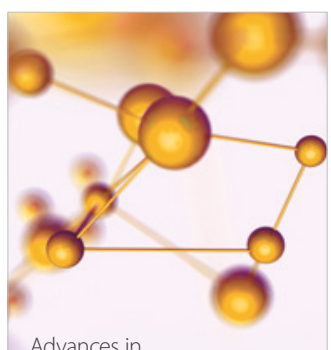

Physical Chemistry
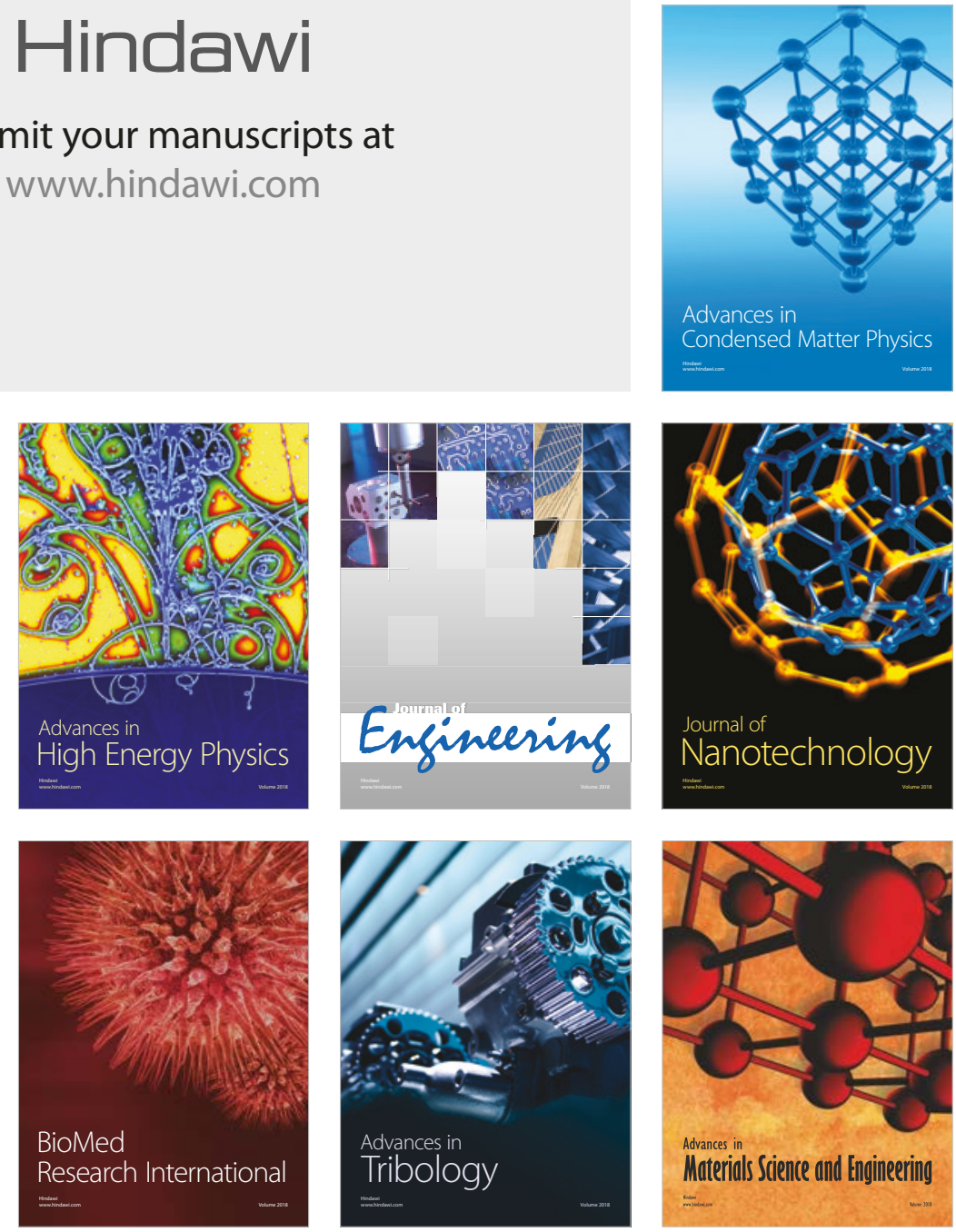